\title{
PROJECT, DESIGN AND MANAGEMENT
}

ISSN: 2683-1597

\section{COMPLEMENTARIEDAD Y ARTICULACIÓN DE LAS METODOLOGÍAS DE PLANIFICACIÓN Y GESTIÓN DE PROYECTOS}

\author{
Santiago Brie \\ Fundación Universitaria Iberoamericana (Argentica) \\ santiagobrie@gmail.com
}

\begin{abstract}
Resumen. Introducción: El objetivo de esta investigación es conocer las estructuras básicas que contienen las metodologías de proyectos y lograr una tipificación que nos permita analizar las posibilidades de complementariedad y articulación de las mismas. Metodología: A partir de la identificación de las principales metodologías de proyectos, se reconocieron tipologías según las organizaciones que las promueven; luego se seleccionaron las más representativas de cada tipo y se realizó una comparación entre los ciclos de vida y los procesos básicos de cada fase dentro del grupo tipificado; posteriormente se desarrollaron tablas síntesis que representan a cada grupo de metodologías y que reflejan el contenido común de cada fase; por último se desarrollan tablas que mostraran los contenidos en cuanto a procesos, componentes e instrumentos. Este proceso permitió una comparación a nivel grupo de metodologías, lo cual hizo posible acceder a conclusiones sobre las posibilidades de complementariedad y articulación. Resultados: el análisis comparativo develó que el grupo de metodologías asociadas a las Agencias de Cooperación cuentan con unas instancias preliminares no presentes en las metodologías propuestas por las asociaciones profesionales; por otro lado, se pudo determinar que las metodologías de las asociaciones profesionales son mucho más complejas y completas en los procesos e instrumental propuesto para las fases de implementación. Discusión: Las metodologías son en muchos aspectos complementarias, una articulación entre ellas permitirá que los profesionales que se desarrollan en la disciplina capitalicen las virtudes y potencialidades de las metodologías que no les son propias, propendiendo a una práctica profesional integral y superadora.
\end{abstract}

Palabras clave: proyectos, metodología, planificación, gestión.

\section{COMPLEMENTARITY AND ARTICULATION OF PLANNING AND PROJECT MANAGEMENT'S METHODOLOGIES}

\footnotetext{
Abstract. Introduction: The objective of this research is to know the basic structures that contain project's methodologies and get a categorization that allows us to analyze the complementarity and articulation of them. Methodology: Starting from the identification of the main project methodologies, categorizations were recognized according to the organizations that promote them; then, the most representative of each type was selected and a comparison was made between the life cycles and the basic processes of each phase
} 
within the identified group; Thereupon, synthesis tables were developed that represent each group of methodologies and reflect the common content of each phase; Finally, tables were developed to show the contents in terms of processes, components and instruments. This process allowed a comparison at the group level of methodologies, which allowed to access to conclusions on the possibilities of complementarity and articulation. Results: the comparative analysis revealed that the group of methodologies associated with the International Cooperation Agencies have some preliminary instances not present in the methodologies proposed by the professional associations; On the other hand, it was possible to determine that the methodologies of the professional associations are much more complex and complete in the processes and instruments proposed for the implementation phases. Discussion: The methodologies are complementary in many aspects, an articulation between them will allow the professionals who develop in the discipline to capitalize on the virtues and potentialities of the methodologies that are not their own, favoring an integral and superior professional practice.

Keywords: Project, methodology, planning, management.

\section{Introducción}

Los estudios orientados hacia el análisis de los proyectos y el establecimiento de metodologías, estándares y pautas destinados al aumento de las probabilidades de éxito en los mismos, han cobrado una creciente relevancia en las últimas décadas. Las aportaciones teóricas y las diversas experiencias han generado enfoques varios en la formulación y gestión de los proyectos. Consecuentemente distintos autores, organizaciones profesionales, e instituciones académicas, abocados al ámbito de los proyectos, estandarizaron estilos de diseño, formulación, gestión y dirección, creando un marco que ha establecido múltiples tendencias en los tópicos antes mencionados. No obstante, existen entre los distintos enfoques grandes puntos de contacto apoyados en la experiencia y documentación de proyectos, y en el desarrollo teórico de una actividad que se ha erigido como una disciplina en sí misma.

Las organizaciones han comprendido que el éxito en la implementación de cambios estratégicos se basa en la formulación y gestión de proyectos y que de la eficacia y eficiencia en ese aspecto depende no sólo el éxito en la adopción de medidas tendientes al cumplimiento de las metas de la estructura organizacional, sino también la primacía frente a un entorno cada vez más competitivo, en el que los proyectos se infiltran en toda la estructura jerárquica de la institución y en todos los procesos: las organizaciones exitosas no sólo procuran el logro de los objetivos de los proyectos, sino también la mejora continua y el desarrollo de recursos humanos idóneos para cada una de las funciones del mismo.

Existe un universo complejo de metodologías sobre proyectos, incluyendo las propuestas por organismos internacionales, agencias de cooperación, asociaciones profesionales, entidades educativas, e incluso por ciertos autores que se han dedicado al desarrollo de las mismas.

A partir de los puntos de coincidencia y disidencia, se analizan las posibilidades de complementación y articulación de estas metodologías para proponer una serie de recomendaciones tendientes, en principio, a identificar aquella que resulte más apta o más apropiada para un tipo de proyecto en particular, o bien para poder integrarlas o articularlas, valiéndose de los aspectos que las hacen más robustas o más flexibles, dependiendo de las necesidades del proyectista.

Se analizaron metodologías diversas provenientes de distintos tipos de organización con la intención de lograr una muestra representativa de la gran variedad de 
estilos y métodos. El criterio específico de selección se encuentra detallado en el apartado Método.

En todas las metodologías analizadas se pudo reconocer una definición de pasos secuenciales organizados en actividades prácticas y el uso de instrumentos de apoyo para estructurar y organizar las operaciones. Este estudio pretende revelar comparativamente qué dedicación brindan las metodologías a cada una de las fases y qué cantidad y complejidad de instrumentos y procesos propone.

\section{Proyectos}

Un proyecto, a diferencia de las actividades laborales cotidianas, son tareas únicas que se realizan para obtener algo que de momento no existe, pudiendo ser un producto o un servicio. Si buscamos obtener un producto o un servicio, es porque deseamos resolver un problema, atender a una necesidad o aprovechar una oportunidad.

Otra característica importante de los proyectos es que se hacen a medida, y por lo tanto no hay dos proyectos idénticos, sencillamente porque no hay dos circunstancias iguales. Las podrá haber parecidas, pero no idénticas. Si deseamos intervenir sobre la realidad tenemos que hacerlo considerando sus particularidades y su complejidad.

De aquí se desprende otra característica de los proyectos y es que, como constituyen intervenciones complejas, necesitamos planificar o programar las acciones, es decir, la forma en que vamos a intervenir.

Los proyectos se diferencian también de las operaciones en curso, por tener unos límites temporales, el proyecto se diseña, se aplica o ejecuta y luego se cierra, deja un sistema funcionando, pero la operación del proyecto culmina. En otras palabras, el sistema funcionando ya no es un proyecto.

La International Project Management Association (IMPA), comprende al proyecto como: "una operación en la cual los recursos humanos, financieros y materiales se organizan de forma novedosa, para realizar un conjunto de tareas, según una especificación definida, con restricciones de costo y plazo, siguiendo un ciclo de vida estándar, para obtener cambios beneficiosos, definidos mediante objetivos cuantitativos y cualitativos" (Reyes y Martínez, 2013, p.21).

\section{Metodologías}

La investigación inicial acerca de las metodologías de proyectos más conocidas, utilizadas y difundidas, permitió reconocer tres grandes grupos de organizaciones fácilmente diferenciables, distinguiendo a:

- $\quad$ Las Agencias de Cooperación Internacional (ACI)

- Los Asociaciones Profesionales de Proyectos (APP)

- Las instituciones académicas

Este trabajo tomó en consideración para el análisis y comparación de las fases del ciclo de vida de los proyectos: sus elementos, procesos e instrumentos constitutivos.

Como premisa básica, se reconoce para el ciclo de vida que implica todo proyecto, una estructura común a todos los métodos, resultado de una división en fases. A pesar de que las características y nomenclatura de estas fases varían según la metodología que sistematiza, en todas ellas subyace la idea de que una fase de Planificación, diseño o formulación, antecede a una fase de ejecución, gestión, dirección o implementación. 


\section{Procesos de Planificación}

La planificación, formulación o diseño de los proyectos, es una instancia compleja que abarca desde el reconocimiento del problema, necesidad o conflicto a resolver; o bien la definición de requisitos establecidos por un comitente (organización o cliente privado) hasta el desarrollo de una propuesta de solución que contenga los aspectos principales necesarios para evaluar y someter a consideración la idoneidad o inviabilidad de tal propuesta.

Lo formulado en las instancias de planeación o diseño, se denomina muchas veces anteproyecto. Durante la formulación, diseño o planificación, no hay materialización, no hay construcción, no se monta nada aún, pero sí se define cómo se realizarán esas fases posteriores, se especifica cómo se materializará o cómo se montará el proyecto cuando comiencen las fases de implementación.

El diseño del proyecto implica un proceso que transita desde lo general hacia lo específico y en el transcurso de ese proceso el proyectista debe recorrer un camino que inicia con la definición de un problema o una necesidad y que culmina con la definición de una solución concreta, el análisis del entorno y el establecimiento de las directrices que lo orientarán hacia la materialización del entregable.

\section{Procesos de Gestión}

Una vez definido qué hacer, cómo hacerlo, dónde hacerlo, cuándo, con quiénes, con qué recursos y para obtener qué resultados, todos elementos especificados (o a veces estimados) en las fases mencionadas anteriormente, se comienza con el proceso de ejecución del proyecto, que implicará unas actividades de gestión, dirección, administración, montaje, construcción, es decir, comienza a ejecutarse lo previamente planificado.

En relación a la forma de nombrar a esta o estas fases, no existe un acuerdo establecido, hay quienes la reconocen como la fase de gestión, dirección, ejecución o implementación, e incluso en ocasiones se utiliza de forma combinada, por ejemplo: fase de gestión y dirección.

Esta situación no se repite en el idioma anglosajón, dado que el término "project management" sintetiza estos procesos necesarios de realizar en la fase de ejecución. Martínez (2016) plantea que no hay una única forma de mencionar las funciones del project management, porque las atribuciones que se utilizan para describir tal función son complementarias: quien se dedica a la implementación o ejecución de proyectos está administrando recursos, dirigiendo personas y gestionando procesos.

\section{Método}

\section{Diseño}

Este estudio está centrado en el análisis de los contenidos de las diversas metodologías sobre proyectos, tomando como objeto de estudio los cuerpos de conocimientos de distintas organizaciones, interpretando los ciclos de vida, los procesos que componen a cada una de las fases del ciclo y los procesos e instrumentos propuestos para cada instancia. 
Se realizó una selección de metodologías según la importancia y difusión; eligiendo aquellas que han sido más utilizadas en sus ámbitos de aplicación o que han marcado tendencias en alguna época en particular.

Del análisis del universo de metodologías y las organizaciones que las promueven, se logró reconocer tres grandes grupos fácilmente diferenciables: uno vinculado a la Agencias de Cooperación Internacional para el desarrollo; otro a las Instituciones o Asociaciones Profesionales de Proyectos, que nuclean y representan a los profesionales que laboran en la disciplina; y otro conformado por las entidades educativas, centrado en las Universidades que abordan la temática de Proyectos.

Para definir la población objetivo de esta investigación se seleccionó un grupo acotado a tres metodologías propuestas por las Agencias de Cooperación Internacional, tres metodologías promovidas por las Asociaciones Profesionales de Proyectos y una metodología que proviene del ámbito educativo, adoptada por tres universidades pertenecientes a la red Funiber.

\section{Participantes}

Se presenta a continuación la selección de las metodologías para cada uno de los tres grupos de organizaciones:

\section{Metodologías de las Agencias de Cooperación Internacional}

Las Agencias de Cooperación Internacional (de aquí en más ACI), son organizaciones que integran un complejo sistema global de entidades, cuyas diversas formas y relaciones constituyen a nivel global el llamado Sistema de la Cooperación Internacional.

La Cooperación Internacional es la relación que se establece entre dos o más países, organismos u organizaciones de la sociedad civil, con el objetivo de alcanzar metas de desarrollo consensuadas.

Las ACI tienen una larga trayectoria en el desarrollo de métodos para el diseño y la formulación de proyectos. Han elaborado un largo listado de metodologías encaminadas a obtener los resultados que se espera para un proyecto de desarrollo. En general cada agencia define su propia metodología a implementar, y en muchas ocasiones se desarrollan nuevos métodos o versiones actualizadas de los ya existentes que implican una evolución con respecto a los que se encuentran en uso (Londoño, 2009).

Para los fines de este trabajo, se analizarán las tres metodologías consideradas como de mayor relevancia, que a su vez han marcado tendencias y que pueden asociarse a distintas épocas en función de su período de mayor utilización. En orden cronológico se pueden ordenar de la siguiente forma:

- Enfoque del Marco Lógico, de la Agencia de Cooperación Internacional de los EEUU (USAID)

- Metodología ZOPP (Planificación de Proyectos Orientada a Objetivos) de la Agencia de Cooperación Internacional Alemana (GTZ)

- $\quad$ Gestión del Ciclo de Proyectos, de la Unión Europea (UE)

El proceso de selección tuvo en consideración los avances ya logrados por Natalia Londoño Vélez, autora del libro Formulación de Proyectos: Enfoques, procesos y herramientas. En este material, se realiza un análisis de 40 metodologías diferentes de formulación o planeación de proyectos desarrolladas por diversas ACI, con el objetivo de 
contribuir a consolidar las políticas y las metodologías de Cooperación Internacional para el Desarrollo de América Latina y Caribe.

Lo que en este caso resulta más interesante del trabajo desarrollado por la autora, son los resultados o conclusiones a los que ha arribado. En palabras de Londoño (2009):

...en todos los modelos metodológicos presentados, la fase de programación es equivalente al diálogo y principios políticos que deben orientar los proyectos de cooperación. La fase de identificación es el primer análisis situacional sobre los participantes, problemas, objetivos y estrategias del proyecto. La formulación es la fase en la cual se consolida el diseño del proyecto, se completa la matriz del marco lógico o de resultados y se verifica la calidad del diseño del proyecto, para tomar la decisión sobre su financiación. (p.3).

Aquí la autora, reconoce puntos en común en cuanto a la forma de referirse a las diferentes fases del proyecto, y también puntos en común en cuanto a lo que implican estas fases iniciales. Esto pudo verse fácilmente reflejado en el trabajo de comparación desarrollado.

\section{Asociaciones Profesionales de Proyectos}

Las Asociaciones Profesionales de Proyectos (desde aquí APP) consideradas más importantes según su cantidad de socios, por la difusión de sus cuerpos de conocimientos y por la aceptabilidad que existe a cerca de las metodologías propuestas, son:

- $\quad$ El Project Management Institute, y su cuerpo de conocimientos en dirección de proyectos conocido como el PMBOK (Project Management Body of Knowledge) actualmente en su 6ta edición, (2017).

- La International Project Management Association y su cuerpo de conocimiento denominado ICB (International Competence Baseline) actualmente en su versión 4 (2016).

- $\quad$ PRINCE2 (Project In Controlled Environment) metodología propuesta por la OGC Office of Government Commerce del Reino Unido, y su cuerpo de conocimientos denominado Managing successful projects with PRINCE2, en su actual 6ta edición, (2017).

El Project Management Institute (PMI) es una organización sin fines de lucro que cuenta con más de medio millón de miembros. Es una de las asociaciones profesionales más grandes del mundo que ofrece certificaciones en dirección de proyectos en 180 países a través del cumplimiento de estándares de dirección.

Los estándares del PMI para la dirección de proyectos, programas y portafolios son reconocidos en la profesión y son sus propios voluntarios del PMI con experiencia en proyectos, los que desarrollan y actualizan estos estándares y proveen un lenguaje común para la dirección de proyectos, programas y portafolios alrededor del mundo.

La International Project Management Association (IPMA) es la primera asociación de gestión de proyectos del mundo. Es una confederación formada por más de 60 asociaciones miembros, con base en Suiza. Las asociaciones que la componen promueven el desarrollo de competencias de gestión de proyectos en sus áreas geográficas de influencia, interactuando con miles de profesionales y desarrollando relaciones con corporaciones, agencias gubernamentales, universidades y colegios, así como organizaciones de formación y consultoría. 
El objetivo de IPMA es desarrollar las competencias profesionales en Dirección de Proyectos, y la certificación IPMA es un medio para alcanzar la excelencia, no es un fin en sí mismo. La certificación está orientada hacia un plan de carrera profesional en Project Management, basado en el desarrollo continuo de competencias en Dirección de Proyectos.

En el caso de PRINCE2, tal es el nombre de la metodología, y en su presentación inicial, no se hizo mención específica de la asociación profesional que la promueve porque es una situación un tanto más compleja. La metodología fue diseñada por el Centro de Informática y la Agencia de Telecomunicaciones del Gobierno del Reino Unido, y es propiedad de la Oficina de Comercio Gubernamental (OGC). Actualmente la metodología es promocionada por AXELOS, una empresa conjunta creada en 2013 por el Gobierno del Reino Unido y la empresa Capita.

A pesar de ser un producto de desarrollo de una dependencia del gobierno, su aplicación trasciende ampliamente las fronteras del Reino Unido, Prince2 ha sido adoptado por distintos organismos gubernamentales de distintos países (Australia, Holanda, Dinamarca, Canadá, entre otros), por empresas multinacionales (DHL, BAT, Barclays, Vodafone, Shell, Unilever, Microsoft, HP, IBM, British Airways, entre otras) y Organizaciones Internacionales (la ONU y sus agencias, el Banco Mundial, entre otras).

Al igual que en el caso de las ACI, es posible encontrar grandes similitudes entre las metodologías propuestas por las distintas Organizaciones vinculadas a la profesión del Director de Proyectos. Coincidencias halladas en las fases propuestas, en los procesos propuestos para cada fase y en los instrumentos utilizados para abordar ciertos temas.

\section{Metodología del ámbito de las Instituciones Educativas}

La enseñanza en proyectos es aplicada mayormente en las instancias de grado y posgrado, y por lo general cada carrera, programa o asignatura elige una metodología de las ya mencionadas en el desarrollo de sus proyectos. Aunque también existe el caso de universidades o cátedras que proponen sus propias metodologías. Para el desarrollo de este trabajo analizaremos la metodología de formulación de proyectos diseñada por los profesores del área de Ingeniería de la Universidad Politécnica de Cataluña, y adaptada por las Universidades de la red Funiber, para el dictado de sus programas de proyecto de posgrado.

Las Universidades que utilizan en sus programas de proyectos esta metodología son:

- Universidad Internacional Iberoamericana de México

- Universidad Internacional Iberoamericana de Puerto Rico

- Universidad Europea del Atlántico

Y los programas en los que es utilizada la metodología son:

- $\quad$ El Máster en Diseño Gestión y Dirección de Proyectos,

- El Máster en Proyectos de Arquitectura y Urbanismo,

- $\quad$ El Máster en Proyectos de Innovación y Producto,

- El Máster en Diseño, Gestión y Dirección de Proyectos de Cooperación Internacional.

La selección de ésta metodología, se justifica no sólo por el uso y la gran aceptación que tiene, sino también porque propone una serie de elementos innovadores: plantea el proceso de planificación de proyectos a través de la práctica, es decir, mediante la 
experiencia, en base a conocimiento empírico, los estudiantes desarrollen un proyecto a partir del reconocimiento de una situación de conflicto, necesidad u oportunidad.

\section{Instrumento}

El análisis documental ha sido el principal recurso constitutivo de la investigación. En todos los casos las metodologías se encuentran plasmadas en documentos, generalmente llamados cuerpos de conocimientos o manuales. En algunos casos también se pueden encontrar publicaciones complementarias promocionadas por estas mismas organizaciones o por autores que son miembro de éstas.

Se realizó un estudio de las últimas versiones editadas por cada una de las organizaciones que promueven las metodologías definidas. Haciendo foco en el reconocimiento de la estructura y composición de cada una de las fases, los procesos o elementos que las componen, y los instrumentos propuestos para el desarrollo de las actividades.

Se utilizaron tablas para facilitar la comparación de la información entre metodologías y cuadros para graficar y sintetizar información.

\section{Análisis de datos}

Para realizar la comparación de datos, y facilitar la interpretación de los mismos, se realizaron los siguientes pasos:

1. Se efectuó una primera comparación entre las metodologías propuestas por cada grupo de organizaciones (las metodologías propuestas por las ACI se compararon entre sí, y lo mismo se hizo con las propuestas por las APP) analizando las diferentes fases y los procesos básicos que las componen.

2. Se realizó una tabla síntesis, que recogiera los elementos comunes de las metodologías de cada grupo de organizaciones (intentando sortear las diferencias en cuanto a la forma de referirse a las fases y procesos).

3. Se confeccionaron tres tablas que, sintetizando la información antes analizada sobre las fases y procesos básicos, reflejara un análisis de los procesos, elementos e instrumentos que cada grupo de metodologías proponía para cada fase.

Esto permitió una comparación más sencilla de las características de las metodologías a nivel grupo, es decir, habiendo previamente reconocido las características que las tipifican.

En cuanto al criterio de selección, para incluir un elemento en la lista de procesos, componentes e instrumentos, la premisa o condición que se estableció es que tal elemento haya estado presente en al menos dos de las tres metodologías comparadas. Es necesario aclarar que en ocasiones la presencia no resulta tan evidente por estar mencionados de forma distinta en uno y otro material, para esto tuvo que realizarse un análisis detallado de las descripciones de cada elemento.

La finalidad de estas tablas síntesis, es poder mostrar un dato estandarizado, referente al contenido de cada grupo de metodologías, para cada fase del proyecto, para facilitar la comparación entre los grupos de metodologías.

Comparación de los Ciclos de Vida con sus fases y procesos básicos, por grupo de organizaciones

A continuación se presenta una comparación de los ciclos de vida de las metodologías de las ACI: 
Tabla 1

Ciclo de Vida de las metodologías de las ACI

\begin{tabular}{|c|c|c|}
\hline Metodología Marco Lógico & Metodología ZOOP & $\begin{array}{l}\text { Metodología Gestión del } \\
\text { Ciclo del Proyecto }\end{array}$ \\
\hline $\begin{array}{l}\text { Fase I de Identificación: } \\
\text { - Análisis de participantes } \\
\text { - Análisis de Problemas } \\
\text { - Análisis de Objetivos } \\
\text { - Análisis de Alternativas }\end{array}$ & $\begin{array}{l}\text { Fase I de Identificación: } \\
\text { - Identificación del problema } \\
\text { central } \\
\text { - Análisis de las causas y } \\
\text { efectos del problema central }\end{array}$ & $\begin{array}{l}\text { Fase I de Programación } \\
\text { - Análisis de orientaciones } \\
\text { políticas } \\
\text { - Principios generales }\end{array}$ \\
\hline $\begin{array}{l}\text { Fase II de Diseño: } \\
\text { - Matriz de Planificación del }\end{array}$ & $\begin{array}{l}\text { - Análisis de Objetıvos } \\
\text { - Análisis de Alternativas }\end{array}$ & $\begin{array}{l}\text { Fase } 11 \text { de Identificación: } \\
\text { - Diagnóstico sobre la idea del } \\
\text { proyecto }\end{array}$ \\
\hline Proyecto & Fase II de Diseño: & \\
\hline $\begin{array}{l}\text { - Programación de } \\
\text { Actividades } \\
\text { - Programación de Recursos } \\
\text { - Factores de Viabilidad } \\
\text { - Documento del Proyecto }\end{array}$ & $\begin{array}{l}\text { - Matriz de Planificación del } \\
\text { Proyecto } \\
\text { - Programación } \\
\text { Actividades } \\
\text { - Programación de Recursos }\end{array}$ & $\begin{array}{l}\text { Fase III de Instrucción o } \\
\text { Formulación: } \\
\text { - Se consolida el diseño del } \\
\text { proyecto }\end{array}$ \\
\hline $\begin{array}{l}\text { Fase III de Ejecución y } \\
\text { Seguimiento: }\end{array}$ & - Documento del Proyecto & - Estudio de Factibilidad \\
\hline $\begin{array}{l}\text { - Plan de ejecución } \\
\text { - Realización operaciones } \\
\text { - Informe de seguimiento }\end{array}$ & $\begin{array}{l}\text { Fase III de Ejecución y } \\
\text { Seguimiento: } \\
\text { - Plan de ejecución } \\
\text { - Realización operaciones } \\
\text { - Informe de seguimiento }\end{array}$ & $\begin{array}{l}\text { Fase V de Ejecución } \\
\text { Seguimiento: } \\
\text { - Utilización de recursos } \\
\text { - Análisis de eficacia } \\
\text { eficiencia }\end{array}$ \\
\hline $\begin{array}{l}\text { impacto, eficacia, eficiencia y } \\
\text { pertinencia }\end{array}$ & $\begin{array}{l}\text { Fase IV de Evaluación: } \\
\text { - Evaluación de viabilidad, } \\
\text { impacto, eficacia, eficiencia y } \\
\text { pertinencia }\end{array}$ & $\begin{array}{l}\text { Fase VI de Evaluación y } \\
\text { Auditoría }\end{array}$ \\
\hline
\end{tabular}

A continuación se presenta una tabla síntesis producto de la comparación de los ciclos de vida de las metodologías de las ACI, reconociendo los procesos básicos de cada fase:

Tabla 2

Cuadro sintesis del Ciclo de Vida de las metodologías de las ACI:

\begin{tabular}{ll}
\hline $\begin{array}{c}\text { Fases de las Metodologías de las } \\
\text { ACI }\end{array}$ & \multicolumn{1}{c}{ Procesos básicos de cada fase } \\
\hline \multirow{2}{*}{ Fase I de Programación } & - Diagnóstico Político \\
& $-\begin{array}{l}\text { Análisis de las Políticas de Cooperación y } \\
\text { Desarrollo }\end{array}$ \\
\hline \multirow{3}{*}{ Fase II de Identificación } & $-\begin{array}{l}\text { Análisis situacional de: participantes, problemas, } \\
\text { objetivos y estrategias }\end{array}$ \\
& - Capacidad Institucional \\
Fase III de Formulación & - Estudios Técnicos de Factibilidad \\
\hline
\end{tabular}




\begin{tabular}{|c|c|}
\hline & $\begin{array}{l}\text { indicadores con líneas base, fuentes de } \\
\text { verificación, calendarios preliminares, } \\
\text { presupuesto y análisis económicos. } \\
\text { - Evaluación del Diseño de la propuesta: valoración } \\
\text { de los criterios de calidad y de los factores de } \\
\text { desarrollo } \\
\text { - Escritura del Documento del Proyecto }\end{array}$ \\
\hline Fase IV de Ejecución & $\begin{array}{ll}- & \text { Plan de Operaciones } \\
\text { - } & \text { Realización de operaciones } \\
- & \text { Informe de avance y de seguimiento } \\
\end{array}$ \\
\hline Fase V de Evaluación & $\begin{array}{l}\text { - Análisis de pertinencia, eficacia, eficiencia, } \\
\text { impacto y viabilidad } \\
\text { - Informe de avance y de seguimiento }\end{array}$ \\
\hline
\end{tabular}

A continuación se presenta una comparación de los ciclos de vida de las metodologías de las APP:

Tabla 3

Ciclo de Vida según las metodologías de las APP

\begin{tabular}{|c|c|c|}
\hline Project Management Institute & $\begin{array}{c}\text { International Project } \\
\text { Management Association }\end{array}$ & PRINCE2 \\
\hline $\begin{array}{l}\text { Fase I de Inicio: } \\
\text { - Acta de Constitución del } \\
\text { Proyecto }\end{array}$ & $\begin{array}{l}\text { Fase I de pre-inversión: } \\
\text { - Evaluación Ex-ante } \\
\text { - Ingeniería Preconceptual }\end{array}$ & $\begin{array}{l}\text { Fase I de Anteproyecto: } \\
\text { - Planificación del escenario } \\
\text { inicial }\end{array}$ \\
\hline $\begin{array}{l}\text { Fase II de Organización y } \\
\text { Preparación: }\end{array}$ & $\begin{array}{l}\text { Fase II de Inversión: } \\
\text { - Ejecución }\end{array}$ & proyecto \\
\hline $\begin{array}{l}\text { - Enunciado del Alcance y } \\
\text { objetivos } \\
\text { - Plan de acción } \\
\text { - Línea base }\end{array}$ & $\begin{array}{l}\text { - Monitoreo } \\
\text { - Informes } \\
\text { - Entrega }\end{array}$ & $\begin{array}{l}\text { Fase II de Escenario Inicial: } \\
\text { - Creación del Plan del } \\
\text { Proyecto (para el escenario 1) } \\
\text { - Revisión del Plan inicial }\end{array}$ \\
\hline $\begin{array}{l}\text { Fase III de Ejecución del } \\
\text { proyecto: } \\
\text { - Avance según Plan de } \\
\text { Acción } \\
\text { - Monitoreo y control } \\
\text { - Aceptación } \\
\text { - Aprobación } \\
\\
\begin{array}{l}\text { Fase IV de Cierre del } \\
\text { proyecto: } \\
\text { - Entrega }\end{array}\end{array}$ & $\begin{array}{l}\text { Fase III de Operación: } \\
\text { - Evaluación de resultados } \\
\text { - Lecciones aprendidas }\end{array}$ & $\begin{array}{l}\text { Fase III de Ejecución del } \\
\text { Proyecto: } \\
\text { - Revisión de la planificación } \\
\text { del escenario } 1 \\
\text { - Ejecución del Plan del } \\
\text { Escenario } 1 \\
\text { - Preparación del Plan del } \\
\text { Escenario } 2 \\
\text { (Repetido tantas veces como } \\
\text { escenarios se propongan). }\end{array}$ \\
\hline & & $\begin{array}{l}\text { Fase (n) de Cierre del } \\
\text { Proyecto: } \\
\text { - Entrega }\end{array}$ \\
\hline
\end{tabular}

A continuación se presenta una tabla síntesis producto de la comparación de los ciclos de vida de las metodologías de las APP, reconociendo los procesos básicos de cada fase: 
Tabla 4

Cuadro Síntesis del Ciclo de Vida de las metodologías de las APP

\begin{tabular}{lll}
\hline $\begin{array}{c}\text { Fases de las Metodologías de } \\
\text { las APP }\end{array}$ & \multicolumn{1}{c}{ Procesos básicos de cada fase } \\
\hline & - & Planificación del Proyecto \\
Fase I: Inicio - Planificación & - Ingeniería preconceptual \\
& - Acta de Constitución del Proyecto \\
& - Plan de Acción \\
\hline \multirow{2}{*}{ Fase II: Ejecución - Operación } & - Avance según Plan de Acción \\
& - Aceptación y aprobación \\
& - Evaluación de Resultados \\
\hline \multirow{2}{*}{ Fase III: Cierre } & - Entrega \\
\hline
\end{tabular}

A continuación se presenta una tabla reconociendo los procesos básicos de cada fase del ciclo de vida según la metodología propuesta por las universidades de la red Funiber:

Tabla 5

Ciclo de Vida según la metodología de las Instituciones Educativas

Metodología de Instituciones Educativas

Fase 1: Identificación del Problema

- Delimitación del problema, alcance, involucrados y requisitos

Fase 2: Anteproyecto

- Definición del sistema solución, los riesgos asociados, recursos humanos y materiales necesarios

- Evaluación de costos de inversión y funcionamiento y proceso de transmisión a futuro equipo gestor.

Fase III: Ingeniería de Detalle

- Estudios de detalle requeridos para la ejecución

Fase IV: Montaje y Construcción

- Ejecución

- Monitoreo y seguimiento

Fase V: Puesta en Marcha y Cesión

- Informes

- Entrega

- Evaluación de resultados

- Lecciones aprendidas

Comparación de procesos, componentes e instrumentos propuestos para cada Fase: 
A continuación se presenta una tabla que contiene las fases comunes al grupo de metodologías representadas por las ACI, donde además se indican los procesos, componentes e instrumentos que constituyen cada fase:

Tabla 6

Procesos, componentes e instrumentos de las ACI.

\begin{tabular}{|c|c|c|}
\hline Fases & Procesos o componentes & Instrumentos \\
\hline Fases de Identificación & Diagnóstico Político & Árbol de Problemas \\
\hline \multirow[t]{9}{*}{ Programación } & Identificación $\quad$ de & Árbol de Efectos \\
\hline & necesidades & Árbol de Causas \\
\hline & Análisis de Problemas & Árbol de Objetivos \\
\hline & Análisis de Objetivos & Matriz de valoración de \\
\hline & Análisis de Involucrados Árbol & poder e interés de los \\
\hline & de Objetivos & involucrados \\
\hline & Identificación de alternativas & Árbol de Acciones \\
\hline & Selección de estrategia & \\
\hline & Definición de Acciones & \\
\hline \multirow[t]{6}{*}{$\begin{array}{l}\text { Fases de Planificación } \\
\text { Formulación }\end{array}$} & $\begin{array}{l}\text { Definición de } \\
\text { Indicadores }\end{array}$ & $\begin{array}{l}\text { Matriz de Planificación del } \\
\text { Proyecto }\end{array}$ \\
\hline & Definición de supuestos & Estructura \\
\hline & Determinación de los medios de & Analítica del Proyecto \\
\hline & seguimiento y control & Matriz Marco Lógico \\
\hline & $\begin{array}{l}\text { Análisis de los factores de } \\
\text { riesgo }\end{array}$ & $\begin{array}{l}\text { Matriz de medios de } \\
\text { verificación }\end{array}$ \\
\hline & & $\begin{array}{l}\text { Técnica de medición de } \\
\text { resultados }\end{array}$ \\
\hline \multirow{3}{*}{$\begin{array}{l}\text { Fases de Ejecución } \\
\text { Evaluación }\end{array}$} & Ejecución de las operaciones & Plan de Operaciones \\
\hline & $\begin{array}{l}\text { Conclusiones evaluación } \\
\text { intermedia }\end{array}$ & $\begin{array}{l}\text { Informe de avance y de } \\
\text { seguimiento }\end{array}$ \\
\hline & $\begin{array}{l}\text { Problemas y acciones } \\
\text { evaluación intermedia } \\
\text { Monitoreo y evaluación }\end{array}$ & $\begin{array}{l}\text { Evaluación Ex post o de } \\
\text { impacto }\end{array}$ \\
\hline
\end{tabular}

A continuación se presenta una tabla que contiene las fases comunes al grupo de metodologías representadas por las APP, donde además se indican los procesos, componentes e instrumentos que constituyen cada fase:

Tabla 7

Procesos, componentes e instrumentos de las APP

\begin{tabular}{|c|c|c|}
\hline Fases & Procesos o componentes & Instrumentos \\
\hline \multirow{2}{*}{ Fases de Planificación: } & Proceso de Inicio del Proyecto & Acta de Constitución del \\
\hline & Proceso de Planificación del & Proyecto (en inglés: charter) \\
\hline \multirow{3}{*}{$\begin{array}{l}\text { - Pre-inversión - } \\
\text { anteproyecto }\end{array}$} & Proyecto & Matriz de análisis de Partes \\
\hline & Plan de gestión del alcance Plan & Interesadas \\
\hline & de gestión del cronograma, & Estructura de División del \\
\hline \multirow{3}{*}{$\begin{array}{l}\text { - Organización y } \\
\text { preparación }\end{array}$} & Plan de gestión de costos Plan & Trabajo \\
\hline & $\begin{array}{l}\text { de gestión de calidad Plan de } \\
\text { gestión de los recursos humanos }\end{array}$ & $\begin{array}{l}\text { Matriz de asignación } \\
\text { responsabilidades }\end{array}$ \\
\hline & $\begin{array}{l}\text { Plan de gestión de las } \\
\text { comunicaciones }\end{array}$ & $\begin{array}{l}\text { Cronograma de tareas } \\
\text { Organigrama }\end{array}$ \\
\hline
\end{tabular}




\begin{tabular}{|c|c|c|}
\hline & $\begin{array}{l}\text { Plan de gestión de riesgos } \\
\text { Plan de gestión de } \\
\text { adquisiciones } \\
\text { Plan de gestión partes } \\
\text { interesadas }\end{array}$ & \\
\hline $\begin{array}{l}\text { Fases de Ejecución: } \\
\text { - Monitoreo y control } \\
\text { - Evaluación } \\
\text { - Cierre }\end{array}$ & $\begin{array}{l}\text { Proceso de Dirección del } \\
\text { Proyecto } \\
\text { Desarrollo y gestión del equipo } \\
\text { de Proyecto } \\
\text { Gestión de las partes } \\
\text { involucradas } \\
\text { Gestión de las comunicaciones } \\
\text { Proceso de Control de Fase del } \\
\text { Proyecto } \\
\text { Seguimiento y control del } \\
\text { trabajo del proyecto } \\
\text { Proceso de Gestión de la } \\
\text { Entrega del Producto } \\
\text { Aseguramiento de la calidad } \\
\text { Control integrado de cambios } \\
\text { Control del cronograma } \\
\text { Control de riesgos } \\
\text { Control de adquisiciones } \\
\text { Administración de contratos }\end{array}$ & $\begin{array}{l}\text { Descomposición de la } \\
\text { Estructura Organizacional } \\
\text { Método Valor ganado } \\
\text { Método PERT } \\
\text { Método CPM } \\
\text { Método ROY } \\
\text { Control de Cronograma } \\
\text { Diagrama de Gantt } \\
\text { Plan de hitos del proyecto } \\
\text { Método del Análisis de la } \\
\text { varianza de Costes } \\
\text { Sistemas de Coste por } \\
\text { Actividad } \\
\text { Técnicas de petición de } \\
\text { oferta } \\
\text { Técnicas de selección de } \\
\text { suministradores } \\
\text { Técnicas para } \\
\text { administración de Contratos } \\
\text { Matriz de evaluación de } \\
\text { riesgos } \\
\text { Técnicas para la gestión de la } \\
\text { calidad } \\
\text { Auditorías de calidad } \\
\text { Clasificación de Costos } \\
\text { Indicadores de evaluación } \\
\text { Económica: VAN, TIR, TD. } \\
\text { Técnicas de valoración del } \\
\text { impacto ambiental }\end{array}$ \\
\hline Fase de Cierre & $\begin{array}{l}\text { Proceso de Gestión de los } \\
\text { Límites de Fase } \\
\text { Validación y control del alcance } \\
\text { Proceso de Cierre del Proyecto } \\
\text { Cierre del proyecto o fase } \\
\text { Cierre de las adquisiciones }\end{array}$ & \\
\hline
\end{tabular}

A continuación se presenta una tabla que contiene las fases de la metodología de las instituciones educativas, donde además se indican los procesos, componentes e instrumentos que constituyen cada fase: 
Tabla 8

Procesos, componentes e instrumentos de la metodología propuesta por las universidades de la red Funiber.

\begin{tabular}{|c|c|c|}
\hline Fases & Procesos o componentes & Instrumentos \\
\hline Fase de Definición & $\begin{array}{l}\text { Identificación del Problema } \\
\text { Reconocimiento de las Causas } \\
\text { Contextualización del problema } \\
\text { Identificación de Limitaciones } \\
\text { Identificación de los } \\
\text { involucrados } \\
\text { Reconocimiento y valoración de } \\
\text { requisitos de los involucrados }\end{array}$ & $\begin{array}{l}\text { Cuadro de definición de } \\
\text { contexto del problema } \\
\text { Matriz de valoración de poder } \\
\text { e interés de los involucrados } \\
\text { Diagramas de Ishikawa } \\
\text { Matriz de valoración de } \\
\text { requisitos } \\
\text { Matriz de contrastación de } \\
\text { requisitos y limitaciones }\end{array}$ \\
\hline Fases de Planificación & $\begin{array}{l}\text { Definición de un sistema que } \\
\text { brinde solución al problema } \\
\text { Desarrollo de los componentes } \\
\text { básicos del sistema } \\
\text { Definición de los recursos } \\
\text { humanos y materiales requeridos } \\
\text { para el desarrollo y posterior } \\
\text { funcionamiento } \\
\text { Identificación de Riesgos } \\
\text { Valoración del impacto al } \\
\text { ambiente } \\
\text { Desarrollo de presupuesto y } \\
\text { cronograma de inversiones } \\
\text { Proceso de cesión del proyecto }\end{array}$ & $\begin{array}{l}\text { Diagramas de flujo } \\
\text { Estructura de División del } \\
\text { Trabajo } \\
\text { Organigrama } \\
\text { Matriz de asignación de } \\
\text { responsabilidades } \\
\text { Estudio de impacto ambiental } \\
\text { simplificado } \\
\text { Técnica de valoración de } \\
\text { riesgos } \\
\text { Cronograma de inversión } \\
\text { Presupuesto }\end{array}$ \\
\hline Fases de Ejecución & \multicolumn{2}{|c|}{$\begin{array}{c}\text { No abordadas en la metodología de las instituciones educativas. } \\
\text { En las maestrías en cuestión, esto está desarrollado en los } \\
\text { Módulos de Gestión y Dirección, donde se propone aplicar lo } \\
\text { propuesto por las APP. }\end{array}$} \\
\hline
\end{tabular}

\section{Resultados}

\section{Resultados del análisis comparativo del Ciclo de Vida de las metodologías de las ACI}

Existe una coincidencia notable entre las fases y los procesos básicos de cada una de las metodologías. A la etapa propia de formulación del proyecto la precede una instancia de programación - identificación, destinada a enmarcar el proyecto dentro de un contexto político institucional. Luego continúa la fase de planificación-formulación donde se desarrollan los componentes básicos del proyecto, determinando las acciones futuras y el rol que tendrá cada uno de los participantes. Posteriormente continúa la etapa de ejecución-seguimiento. En ella no se aportan grandes conceptos o herramientas ni se especifica la misma división detallada de procesos que sí se expone en las fases iniciales, más bien se especifica cómo desarrollar el seguimiento de las actividades, pero no cómo se ejecuta. Y por último existe una fase de evaluación en la que se analizan los aspectos de eficacia, eficiencia, impacto, viabilidad y pertinencia.

Estos resultados están en línea con los que propone Londoño (2009), quien señala: 
Si bien, cada agencia presenta diferencias en el número y denominación de las fases para estructurar un proyecto, así como tenues variaciones según la filosofía institucional y el énfasis en uno $u$ otro enfoque metodológico, el significado, así como los procesos que componen cada fase, son casi idénticos y homologables. Sustancialmente pueden señalarse tres fases comunes y centrales en la construcción de un proyecto: planeación, implementación y evaluación. (p. 27).

Esto puede explicarse en el sentido de que este tipo de proyectos tiende siempre a lograr el desarrollo y beneficio de un determinado grupo de personas, con el compromiso y participación de diferentes actores, y con enfoques y análisis transversales que propendan a la equidad en una comunidad, como lo son entre otras, las perspectivas medioambientales y de género.

Resultados del análisis comparativo del Ciclo de Vida de las metodologías de las APP:

Del análisis del ciclo de vida, las fases y procesos básicos de las APP, también es posible reconocer que existen mayores coincidencias que diferencias, iniciando generalmente con una fase de inicio-planificación-formulación que no requiere de instancias previas, donde las tareas están abocadas a desarrollar el plan de acción o el plan del proyecto. Lo continúa una fase de ejecución-seguimiento, donde se realiza lo definido en la instancia inicial, controlando, monitoreando y evaluando constantemente los procesos y resultados. Posteriormente se desarrolla una fase de cierre, en la que se realizan las entregas de los productos desarrollados y la finalización de contratos y demás relaciones contractuales.

Resultados del análisis de procesos, elementos e instrumentos de las metodologías propuestas por las ACI:

De este análisis pudo deducirse que las metodologías de las ACI dedican mucho tiempo y esfuerzo a las definiciones preliminares que se abocan a determinar la comunidad o grupo beneficiado, los problemas centrales, las percepciones de los mismos de parte de los actores involucrados, las estrategias y alternativas de acción, esto es, una serie de procesos que preceden a la determinación del plan de acción. Al mismo tiempo, los procesos, elementos e instrumentos que se proponen para las fases de ejecución y seguimiento, tienen mucho menos desarrollo, e incluso atienden casi exclusivamente la función de control sobre la aplicación de las actividades, pero sin definir cómo aplicar o desarrollar tales actividades.

Resultados del análisis de procesos, elementos e instrumentos de las metodologías propuestas por las APP:

En contraposición con lo verificado en las metodologías de las ACI, en el caso de las metodologías propias de las APP se puede advertir que los procesos inician con la definición del plan de acción, es decir, las definiciones preliminares sobre qué se pretende hacer parecen estar ya definidas y no ser parte de los procesos de planificación. Esto queda claramente representado en el caso del Ciclo de Vida del PMI donde se señala que el inicio del proyecto se desprende de la directiva de la dirección de la empresa sobre un requisito específico.

Por otro lado, la cantidad de procesos e instrumentos propuestos para las fases de ejecución y seguimiento son notablemente más numerosos y complejos, lo que denota una mayor dedicación y preocupación por estas instancias. 
Resultados del análisis de procesos, elementos e instrumentos de las metodologías propuestas por las Instituciones Educativas:

En el caso de esta metodología, se puede observar que tanto en la definición de las fases de su ciclo de vida como en los instrumentos propuestos para cada instancia, existe una coincidencia evidente con las metodologías propuestas por las ACI. En relación a las fases de ejecución y seguimiento, no hay elementos para realizar la comparación ya que esta metodología está abocada a los procesos de planificación y formulación pero no a los de gestión y ejecución. Tales contenidos son abordados en los programas de maestría analizados, en módulos distintos, donde se propone la aplicación de los instrumentos y procesos desarrollados por IPMA y el PMI.

Resultados que permite la comparación de los procesos, elementos e instrumentos de los tres grupos de organizaciones:

Comparando los resultados obtenidos en las tres tablas, podemos observar que hay primero una diferencia en cuanto a las fases en que se estructuran unas y otras metodologías, teniendo mucho en común las propuestas por las ACI y la metodología del ámbito educativo, pero teniendo grandes diferencias con las propuestas por las APP.

Las primeras (ACI e instituciones educativas) se centran en "qué hacer", y demuestran una gran dedicación en las instancias previas de definición o programación, donde se pretende establecer un marco de acción y se intenta definir un escenario consensuado con actores que serán beneficiarios o que estarán influidos por la intervención; mientras que las segundas (APP) se concentran en el "cómo realizarlo", teniendo previamente definido y determinado lo que se pretende conseguir.

Esto queda claramente reflejado en los procesos, elementos e instrumentos propuestos para cada fase por cada grupo de metodologías: se observa una superioridad en cantidad y complejidad de los factores analizados en las fases preliminares para las ACI y la metodología de las instituciones educativas, mientras que tal superioridad la hallamos en el tratamiento que las metodologías de las APP hacen de las fases de gestión y ejecución.

\section{Discusión y conclusiones}

\section{Conclusiones de los resultados de la investigación}

En función de los resultados obtenidos por la investigación, y aclarando que esto no intenta ser representativo de la totalidad de los casos existentes, sino a la población definida como objeto de estudio de esta investigación, podemos establecer las siguientes conclusiones:

- Las metodologías de proyectos propuestas por las ACI están centradas en los procesos iniciales, atendiendo a la definición preliminar y programación de las acciones, pero soslayando procesos fundamentales de la ejecución de los proyectos.

- Las metodologías de proyectos propuestas por las APP tienen un menor desarrollo de las instancias preliminares en comparación con las propuestas por las ACI, pero los aspectos referentes a las instancias de ejecución del proyecto están mucho más desarrollados, con una batería muy completa de instrumentos destinados a la aplicación y ejecución de las actividades. 
- La metodología propuesta por las universidades de la red Funiber, tal como la conciben los programas de proyectos, está abocada sólo a las instancias de planificación y tiene grandes puntos de coincidencia con las metodologías propuestas por las ACI, planteando puntos de partida diferentes, pudiendo también ser adaptable al desarrollo de proyectos en el ámbito privado.

- Las metodologías propuestas por las ACI son recomendables para proyectos con intenciones definidas, vinculadas a promover el desarrollo de una comunidad o una región, pero muchas veces con incertidumbre en relación a la forma de obtener o lograr dichos resultados.

- Las metodologías propuestas por las APP son recomendables para proyectos que tienen definido el producto o servicio que pretende lograr u ofrecer, y que requieren de la definición de los procesos para lograr dichos resultados.

- A pesar de tener en los tres grupos de metodologías puntos de partida distintos, estas son esencialmente complementarias; en los casos en los que se desarrollan proyectos por parte de las ACI, para el desarrollo de las fases de ejecución es posible valerse de los procesos, elementos e instrumentos propuestos por las APP.

- También es posible una complementariedad de metodologías cuando las empresas (que en general utilizan las metodologías de las APP) desarrollan proyectos con impacto en la comunidad o en el ambiente. Para estos casos, valerse de los procesos e instrumentos propuestos por las ACI puede resultar fundamental para conseguir el éxito del proyecto.

\section{Representación gráfica de los resultados}

En el siguiente gráfico quedan reflejadas las fases adoptadas por cada grupo de metodologías.

Metodologías de las ACI:

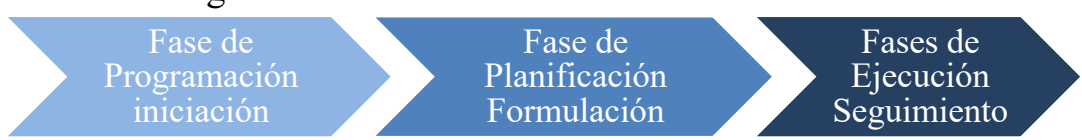

Metodologías de las APP:

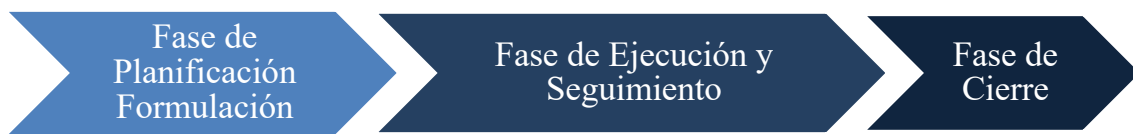

Metodología de las IE:

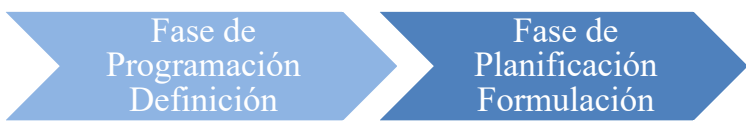

Figura 1. Fases por cada grupo de metodologías

A continuación se expone un gráfico que representa la dedicación según cantidad de procesos, elementos e instrumentos propuestos para cada fase, diferenciado por grupos de metodologías: 


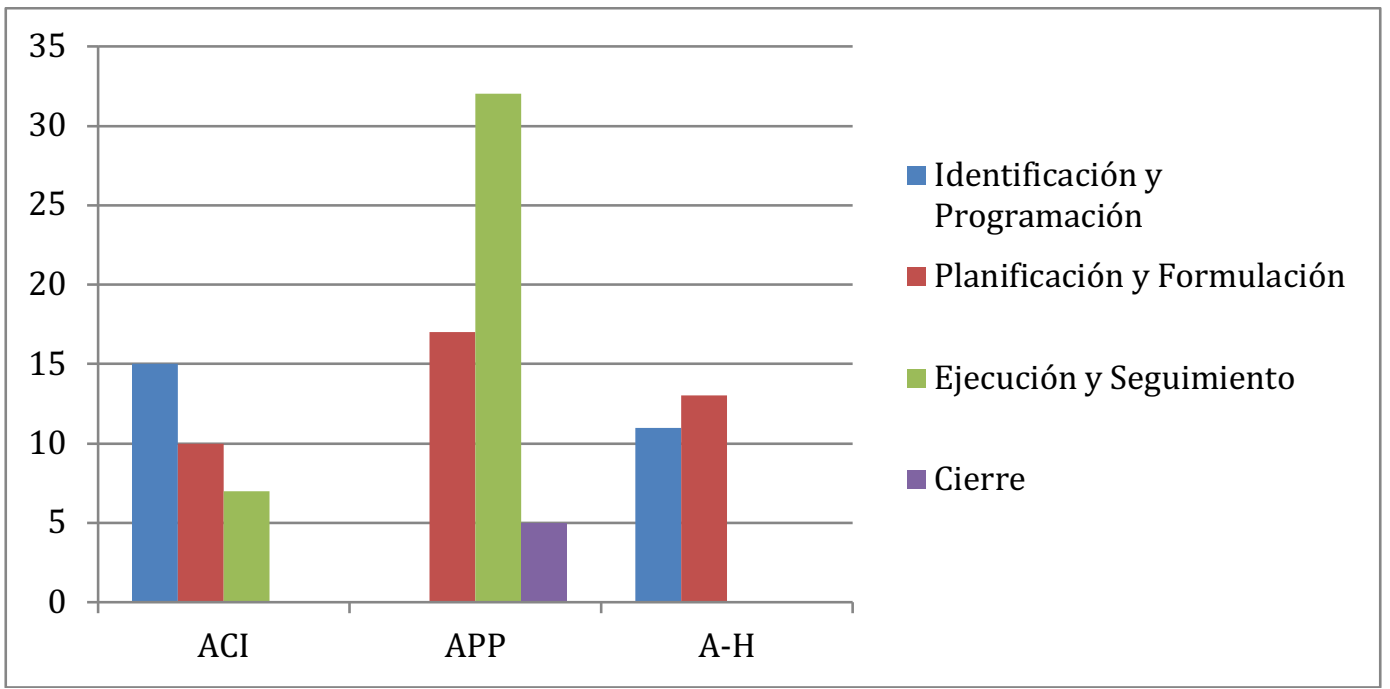

Figura 2. Procesos, elementos e instrumentos de cada fase en cada grupo de metodologías.

\section{Reflexión del autor}

Trabajo en docencia universitaria de grado aplicando metodologías propias de las ACI y en docencia de posgrado donde aplicamos la metodología de las universidades de la red Funiber; también soy miembro de la Asociación Argentina de Gestión de Proyectos en la que promovemos el uso de las metodologías desarrolladas por las APP. En base a esta experiencia puedo decir, que a pesar de ser claras las diferencias entre los tipos de metodologías y su aplicabilidad, esto no parece ser evidente para profesionales del área. Es común que docentes del ámbito universitarios desconozcan los instrumentos y recursos que aportan las metodologías de las APP para la ejecución de los proyectos, y en el mismo sentido, sorprende a veces el desinterés que existe incluso de parte de profesionales de las APP, sobre los instrumentos y aplicabilidad de las metodologías de las ACI.

Es frecuente entonces que se desaproveche este potencial que nos provee la integración disciplinar en tanto muchas veces quienes están abocados a la planificación desconocen las virtudes de las herramientas de gestión, y quienes se dedican a la ejecución, no tienen en consideración los beneficios de las metodologías de planificación.

\section{Conclusiones generales}

La comparación de los contenidos y gráficos nos permite reafirmar la tendencia observada acerca del objeto de estudio o trabajo de unas y otras organizaciones. En el caso de las metodologías de las ACI, el mayor nivel de detalle está centrado en los procesos iniciales de definición, planificación y formulación, correspondientes a las tareas de diseño de los profesionales involucrados; mientras que el material que disponen y promueven las APP, presentan un desarrollo más exhaustivo en las fases de ejecución, concernientes a las tareas de gestión y dirección de los profesionales.

Esto se debe fundamentalmente a los motivos que llevan en uno y otro caso a desarrollar los proyectos. Para el caso de los proyectos de Desarrollo, promovidos por las ACI, la motivación es beneficiar a una comunidad o grupo de personas, por lo que no está 
definida la forma o a través de qué producto o servicio se concretará, y por esto estas fases iniciales son más extensas y requieren de más tiempo, herramientas y métodos particulares para tal definición; en cambio en el tipo de proyectos que se aborda desde las APP, muchas veces ya se cuenta con el objetivo a lograr e incluso con la definición del producto a conseguir o servicio a ofrecer, predeterminados por los directivos de la organización o por los clientes de la empresa. En estos casos se requiere de menos tiempo y recursos para las definiciones más básicas e iniciales del proyecto.

En los proyectos desarrollados por una empresa, que continúan la lógica de las metodologías de las APP, es común que el dueño del proyecto y el ejecutor del mismo, sea la propia empresa, por tanto, las metodologías proponen una batería de instrumentos operativos para los procesos de gestión, administración y dirección ; por el contrario, en los proyectos promovidos por las ACI, es común que el dueño y ejecutor del proyecto sea una ONG, o institución u organización de la sociedad civil a la que se asesora, acompaña y a la que en ocasiones se le hace un seguimiento, por tanto los instrumentos que proponen para estas instancias apuntan al control más que a la ejecución de las operaciones.

Esto queda evidenciado en lo postulado en el manual Metodología del marco lógico para la planificación, el seguimiento y la evaluación de proyectos y programas, del Instituto Latinoamericano y del Caribe de Planificación Económica y Social. En este documento que dedica un $95 \%$ de su contenido a los aspectos de Diseño y formulación, los autores Ortegón, Pacheco y Prieto (2005) afirman:

El Seguimiento o Monitoreo, se efectúa durante la etapa de ejecución de un proyecto y no en otras etapas del ciclo del proyecto. Es un procedimiento sistemático empleado para comprobar la eficiencia y efectividad del proceso de ejecución de un proyecto para identificar los logros y debilidades y recomendar medidas correctivas para optimizar los resultados deseados. (p.47)

La metodología Marco Lógico propone como elemento integrador entre la Fase de planificación y la de ejecución, el Plan de Monitoreo y Evaluación. Al respecto Oregón et al (2005) afirma: "Vale la pena indicar que sin el establecimiento de un buen plan de MyE, el Gerente de Proyecto no tiene el elemento básico de gestión en sus manos” (p 50).

\section{Referencias}

AEIPRO-IPMA. (2009). NBC-Bases para la Competencia en Dirección de Proyectos. Valencia: Editorial UPV.

AXELOS (2009). Managing Successful Projects with PRINCE2. London: Editorial Van Haren Publishing.

AXELOS (2017) Managing Successful Projects with PRINCE2® 2017 Edition First Edition, Second Impression.

Blasco, J. (2003). Los proyectos de sistemas artificiales: el proyectar y lo proyectado. Barcelona: Ediciones UPC.

Camacho H. Et. Al (1999). El enfoque del marco lógico: 10 casos práctico. Madrid: Fundación CIDEAL y Acciones de Desarrollo y Cooperación. 
Cejas, C.; Olaviaga, S.; Kremer, P. (2006): Manual para la formulación de proyectos de organizaciones comunitarias. Buenos Aires: CIPPEC

Comisión Económica para América Latina y el Caribe -CE- PAL. Instituto Latinoamericano y del Caribe de Planificación Económica y Social -ILPES. (2005) Metodología del Marco Lógico para la planificación, el seguimiento y la evaluación de proyectos y programas.

FUNIBER. (2017) Material del Módulo de Diseño de la Maestría en Proyectos. Barcelona: Fundación Universitaria Iberoamericana

Gómez, M., Sinz, H. (2003). El ciclo del proyecto de cooperación al desarrollo. CIDEAL. P. 221.

IPMA International Project Management Association (2015). NCB4 Competence Baseline. International Project Management Association.

Londoño Vélez, N. (2009). Formulación de Proyectos: Enfoques, procesos y Herramientas. Escuela Latinoamericana de Cooperación y Desarrollo.

Ministerio de Asuntos Exteriores de España. Secretaría de Estado de Cooperación Internacional y para Ibero América (1997). SECIPI: Metodología de evaluación de la cooperación española. Madrid.

Osorio, S., Zamora, V., Jimenez, M. y Macias, H. (2015). Guía Metodológica para el Diseño de Proyectos de Intervención de la Práctica Educativa. Revista de Docencia e Investigación Educativa, 1 (1), 39-48. Retrieved from https://dialnet.unirioja.es/servlet/articulo? codigo $=6367046$

Planificación de Proyectos Orientada a Objetivos (ZOPP). (1998) Deutsche Gesellschaft für Technische Zusammenarbeit-GTZ. GmbH, Eschborn.

PMI, Project Management Institute. (2017). Guía de los Fundamentos para la Dirección de Proyectos (Guía del PMBOK) (6 ${ }^{\mathrm{a}}$ Ed.). Pennsylvania: Project Management Institute.

Reyes, J. E., y Martínez Almela, J. (2013). Procesos de Proyectos y Competencias en Dirección de Proyectos. Valencia: Editorial UPV.

Fecha de recepción: 16/05/2019

Fecha de revisión: 29/10/2019

Fecha de aceptación: 03/05/2020 